\title{
Study of Weakly nonlinear Mass transport in Newtonian Fluid with Applied Magnetic Field under Concentration/Gravity modulation
}

https://doi.org/10.1515/nleng-2018-0058

Received March 23, 2018; revised June 26, 2018; accepted August 3, 2018.

\begin{abstract}
In the present paper, a weakly nonlinear stability analysis is used to analyze the effect of time-periodic concentration/gravity modulation on mass transport. We have considered an infinite horizontal fluid layer with constant applied magnetic flux salted from above, subjected to an imposed time-periodic boundary concentration (ITBC) or gravity modulation (ITGM). In the case of ITBC, the concentration gradient between the plates of the fluid layer consists of a steady part and a time-dependent oscillatory part. The concentration of both walls is modulated. In the case of ITGM, the gravity field consists of two parts: a constant part and an externally imposed time periodic part, which can be realized by oscillating the fluid layer. We have expanded the infinitesimal disturbances in terms of power series of an amplitude of modulation, which is assumed to be small. Ginzburg-Landau equation is derived for finding the rate of mass transfer. Effect of various parameters on the mass transport is also discussed. It is found that the mass transport can be controlled by suitably adjusting the frequency and amplitude of modulation.
\end{abstract}

Keywords: Newtonian fluid, Applied magnetic field, Concentration modulation, Gravity modulation, Ginzburg Landau equation

\footnotetext{
*Corresponding Author: Anand Kumar, Department of Mathematics, Central University of Rajasthan, Kishangarh-305817, India, E-mail: aanandbhu@gmail.com

Om Prakash Keshri, Department of Mathematics, Central University of Rajasthan, Kishangarh-305817, India

Vinod K. Gupta, Department of Mathematics, DIT University, Dehradun-248009, India
}

\section{Introduction}

The transport phenomena such as heat and mass transfer, play a vitally important role in human life. Convection and diffusion are responsible for temperature fluctuations and transport of pollution in air, water and soil. The ability to understand, predict and control to the transport phenomena is essential for many industrial applications such as aerodynamic shape design, oil recovery from an underground reservoir or multiphase/multicomponent flows in furnaces, heat exchange, and chemical reactors.

Many researchers have investigated the effect of temperature/gravity modulation on the onset of convection in a viscous fluid layer due to its wide range of applications in industries as well as technological areas such as biotechnology, microelectronics, nanotechnology and polymer science. The subject of mass transfer studies relative motion of some chemical species concerning other, driven by a concentration gradient. The linear theory of mass transfer has three basic features characterized as the mass transfer rate does not depend on the direction of mass exchange between phases, the Sherwood number does not depend on concentration and the mass transfer does not influence the hydrodynamics. The development of mass transfer theory closely follows as in heat transfer with the pioneering works of Lewis and Whitman [1] and Sherwood [2]. Bird et al. [3] jointly studies heat transfer, mass transfer, and momentum transfer and considered as a new discipline at that time.

Chandrasekhar [4] and Bejan [5] both provides a comprehensive review of the fundamentals of stability problem with uniform heating in porous media. The effect of modulation on the stability of the flow between rotating cylinder was investigated experimentally by Donnelly [6]. Venezian [7] investigated the thermal analog of Donnelly's experiment, using free-free surfaces and compared his results with them. Rosenblat and Herbert [8] have investigated the linear stability problem for free-free surfaces using low-frequency modulation and found an asymptotic solution. Researchers have solved many problems concerning how a time-dependent boundary temperature af- 
fect the onset of Rayleigh-Bénard convection. Davis [9] has reviewed most of the investigations relevant to these problems. Rosenblat and Tanaka [10] have used Galerkin procedure to study the linear problem for more realistic boundary conditions. Besides investigating the linear stability, Roppo et al. [11] have also carried out the weakly nonlinear analysis of the problem. A similar problem has been considered earlier by Gershuni and Zhukhovitskii [12] for a temperature profile obeying the rectangular law. Yih and Li [13] have investigated the formation of convective cells in a fluid between two horizontal rigid boundaries with time-periodic temperature distribution using Floquet theory.

Finucane and Kelly [14] have carried out an analytical experimental investigation on the results of Rosenblat and Herbert [8]. Aniss et al. [15] have worked out a linear problem of the convection parametric instability in the case of a Newtonian fluid confined in a Hele-Shaw cell and subjected to the periodic vertical motion. In their asymptotic analysis, they have investigated the influence of the gravity modulation on the instability. Further, Bhatia and Bhadauria [16, 17] and Bhadauria and Bhatia [18] have studied the linear stability problem for sawtooth, stepfunction, and day-night profiles. Recently, Bhadauria et al. [19] have investigated the local nonlinear stability analysis of modulated double-diffusive stationary convection for coupled stress liquid. Kumar et al. [20] have shown the effect of gravity modulation on double diffusive convection of couple stress liquid and found that on increasing $P r$, $R a_{S}$, Le and Couple stress parameter increases the rate of heat and mass transfer.

The first investigation on the effect of gravity modulation of fluid layer heated from below was reported by Gersho and Sani [21]. Gershuni et al. [22] and Kamotini et al. [23] have applied a perturbation technique to analyze horizontal gravity modulation phenomena. Biringen and his coworkers $[24,25]$ have used the finite difference method to study the gravity modulation effect in 2D and 3D rectangular cavities under normal-gravity and zero gravity conditions. Experiments on the response of Rayleigh-Bénard convection to gravity modulation were carried out by Rogrers et al. [26]. Wadih and Roux [27] presented a study on the instability of the convection in an infinitely long cylinder with gravity modulation oscillating along the vertical axis. Clever et al. [28] used Galerkin method after different range of parameters for the oscillatory convection in a gravitationally modulated fluid layer.

All the above study did not describe the effect of magnetic field. Although the magnetic field has the property that controls the flow formation externally and uses in many experimental and scientific types of equipment. Many re- searchers considered the study of flow formation in different boundary condition under the inflation of magnetic field [29-37]. Srivastava et al. [38] investigate Soret effect on magneto-convection fluid flow in an anisotropic porous layer. Gupta et al. [39] also reported the effect of magnetic field on chaos in couple stress liquid in the porous layer. Swamy [40] have examined the effect of gravity modulation on dielectric fluid. Recently, Gupta et al. [41] and Gupta [42] presented the weakly nonlinear study of mass transport in a rotating fluid layer in presence of concentration modulation.

To the best of authors' knowledge, till date, there is no nonlinear study available which performed the effect of concentration modulation in presence of a magnetic field. It is with these motives that a weakly nonlinear analysis in an infinite horizontal fluid layer with the applied magnetic field has been made under concentration/ gravity modulation. Using the Ginzburg - Landau equation the effect of an applied magnetic field on mass transport is also studied. In this paper, we have investigated the effect of concentration/ gravity modulation on the convective mass transport of viscous incompressible Newtonian fluid between two horizontal parallel plates. The amplitude and frequency of the concentration and gravity modulations are externally controlled parameters, and hence the rate of mass transfer can be delayed or advanced by proper tuning of these parameters. The problem has potential application in achieving major enhancement of concentration and momentum in the material processing and related areas.

\section{Mathematical Formulation}

We consider viscous, incompressible and electrically conducting fluid confined between two parallel infinite horizontal plates $z=0$ and $z=d$ at a distance $d$ apart salted from above. We have chosen a Cartesian frame of reference in which the origin lies on the lower plate and the $z$-axis vertically upwards. In the present study, we have not considered the interface deformation, and the densities of the fluid and melt are assumed equal so that convection due to density variation neglected and only solutal convection taken in account. Coriell et al. [43] and Hurle et al. [44] reported some conditions for unmodulated problem under which the effect of thermally driven convection and interface deformation are negligible. Wheeler et al. [45] have considered the Navier-Stokes equations and conservation equation for solutal convection in their study. They have neglected the convection due to a density change and considered only solutal convection. The Boussinesq approxi- 
mation is employed which assumes that the density varies linearly with solute concentration and that is included only in body force term in the Navier-Stokes equations. A uniform magnetic field of strength $B_{0}$ is applied perpendicular to both the plates. Under these assumptions the governing equations can be written as (Gupta et al. [39], Gupta et al. [41] and Wheeler et al.[45]):

$$
\begin{gathered}
\nabla \cdot \vec{q}=0 \\
\rho_{0}\left[\frac{\partial \vec{q}}{\partial t}+(\vec{q} \cdot \nabla) \vec{q}\right]=-\nabla \mathrm{p}+\rho \vec{g}+\mu \nabla^{2} \vec{q}-\sigma \mu_{e}{ }^{2} B_{0}{ }^{2} \vec{q} \\
\frac{\partial S}{\partial t}+(\vec{q} \cdot \nabla) S=\kappa_{S} \nabla^{2} S \\
\rho=\rho_{0}\left[1+\beta_{S}\left(S-S_{0}\right)\right]
\end{gathered}
$$

where $\vec{q}$ is the velocity, $\mathrm{p}$ the pressure, $\rho$ is the density, $g$ is the gravitational acceleration, $\mu$ is the viscosity, $\sigma$ is the electrical conductivity, $\mu_{e}$ is magnetic permeability, $B_{0}$ is the strength of applied magnetic field, $\mathrm{S}$ is the solute concentration, $\kappa_{s}$ is the mass diffusivity of the fluid, $\beta_{s}$ is the mass expansion coefficient.

\subsection{Time-periodic Concentration Modulation}

In this section, we present the concentration modulation problem. The external driving force is modulated harmonically in time by varying the concentrations of lower and upper horizontal boundary. Accordingly, we have considered the concentration modulation at the boundary

$$
\begin{gathered}
S=S_{0}-\frac{\Delta S}{2}\left[1-\epsilon^{2} \delta_{1} \cos (\Omega t)\right] \quad \text { at } \quad z=0 \\
S=S_{0}+\frac{\Delta S}{2}\left[1+\epsilon^{2} \delta_{1} \cos (\Omega t+\varphi)\right] \quad \text { at } \quad z=d
\end{gathered}
$$

where $\epsilon$ represent small amplitude of modulation, $\Omega$ the frequency of modulation, $\varphi$ the phase angle. We consider three types of concentration modulation, viz., Case(a) : symmetry (in-phase modulation (IPM), $\varphi=0$ ), Case(b) : asymmetry (out-phase modulation (OPM), $\varphi=\pi$ ) and

Case(c) : only upper wall concentration is modulated while the lower one is held at constant concentration (UPM) $(\varphi=$ $-i \propto)$

\subsection{Basic State}

The basic state of the fluid is quiescent and is given by

$$
\begin{aligned}
& \overrightarrow{q_{b}}=(0,0,0), \quad p=p_{b}(z, t), \quad S=S_{b}(z, t), \\
& \text { and } \rho=\rho_{b}(z, t)
\end{aligned}
$$

Which satisfy the following equations

$$
\begin{gathered}
\frac{\partial S_{b}}{\partial t}=\kappa_{s} \frac{\partial^{2} S_{b}}{\partial z^{2}} \\
\frac{\partial p_{b}}{\partial z}=\rho_{b} g
\end{gathered}
$$

and pressure $p_{b}(z, t)$ balances the buoyancy force.

The solution of Eq. (8) subject to the boundary conditions (5) and (6) is given as

$$
\frac{\partial S_{b}}{\partial z}=\frac{\Delta S}{d}\left[1+\epsilon^{2} \delta_{1} f(z, t)\right]
$$

where

$$
\begin{aligned}
& A(\lambda)=\frac{\lambda}{2}\left(\frac{e^{-i \varphi}-e^{-\lambda}}{e^{\lambda}-e^{-\lambda}}\right), \quad \lambda^{2}=\frac{-i \Omega d^{2}}{\kappa_{s}}, \\
& f(z, t)=\operatorname{Re}\left[\left(A(\lambda) e^{\frac{\lambda z}{d}}+A(-\lambda) e^{\frac{-\lambda z}{d}}\right) e^{-i \Omega t}\right]
\end{aligned}
$$

and Re stand for real part.

Now, we consider the infinitesimal perturbations techniques to the basic state solution in the form

$\vec{q}=\vec{q}_{b}+\vec{q}^{\prime}, \quad S=S_{b}+S^{\prime}, \quad p=p_{b}+p^{\prime} \quad$ and $\quad \rho=\rho_{b}+\rho^{\prime}$

where prime indicates that the quantities at perturbed state.

Using Eq. (11) into Eq. (1)-(4) and using the basic state solution, we obtained the governing equations in perturbed form as follows

$$
\rho_{0}\left[\frac{\partial \overrightarrow{q^{\prime}}}{\partial t}+\left(\overrightarrow{q^{\prime}} \cdot \nabla \overrightarrow{q^{\prime}}\right)\right]=-\nabla p^{\prime}+\rho^{\prime} \vec{g}+\mu \nabla^{2} \overrightarrow{q^{\prime}}-\sigma \mu_{e}{ }^{2} B_{0}{ }^{2} \overrightarrow{q^{\prime}}
$$

$$
\begin{gathered}
{\left[\frac{\partial S^{\prime}}{\partial t}+\left(\overrightarrow{q^{\prime}} \cdot \nabla\right) S^{\prime}\right]+w^{\prime} \frac{\partial S_{b}}{\partial z}=\kappa_{s} \nabla^{2} S^{\prime}} \\
\rho^{\prime}=\rho_{0} \beta_{s} S^{\prime}
\end{gathered}
$$

In order to eliminate the pressure term from Eq. (12), we take the curl of Eq. (12). Since, We are assuming all physical quantities to be independent of $y$, due to the study being restricted to rolls with the $y$-coordinate as their axis, introducing the stream function as $u=\frac{\partial \psi}{\partial z}, \quad w=-\frac{\partial \psi}{\partial x}$. Eqs. (12)-(13) may be written in dimensionless form by using following non-dimensional quantities

$$
\begin{aligned}
& (x, y, z)=\left(x^{\star}, y^{\star}, z^{\star}\right) d, \quad t=\left(t^{\star}\right) \frac{d^{2}}{\kappa_{S}}, \quad(S)=S^{\star} \Delta S \\
& \text { and } \psi=\left(\psi^{\star}\right) \kappa_{S},
\end{aligned}
$$


as (for simplicity we drop the asterisks):

$$
\frac{1}{S c} \frac{\partial}{\partial t}\left(\nabla^{2} \psi\right)=\operatorname{Ras} \frac{\partial S}{\partial x}+\nabla^{4} \psi-H a^{2} \nabla^{2} \psi+\frac{1}{S c} \frac{\partial\left(\psi, \nabla^{2} \psi\right)}{\partial(x, z)}
$$

$$
\frac{\partial S}{\partial t}-\left(1+f \epsilon^{2} \delta_{1}\right) \frac{\partial \psi}{\partial x}=\nabla^{2} S+\frac{\partial(\psi, S)}{\partial(x, z)}
$$

where $f=\operatorname{Re}\left[A(\lambda) e^{\lambda z}+A(-\lambda) e^{-\lambda z}\right] e^{-i \omega t}$. $S c=\frac{v}{\kappa_{s}}$ is the Schmidt number ( ratio of diffusion in momentum to diffusion in solute ), $\operatorname{Ras}=\frac{\alpha_{s} g(\Delta S) d^{3}}{\nu \kappa_{s}}$ is the Solute Rayleigh number, $\mathrm{Ha}=\mu_{e} B_{0} d \sqrt{\frac{\sigma}{v}}$ is the Hartmann number.

\section{Non-linear Stability Analysis}

we will consider the slow time variations at the scale $\tau=$ $\epsilon^{2} t$, then Eq. (15) and (16) becomes

$$
\begin{gathered}
{\left[\frac{\epsilon^{2}}{S c} \frac{\partial}{\partial \tau}\left(\nabla^{2}\right)-\nabla^{4}+H a^{2} \nabla^{2}\right] \psi-R a s \frac{\partial S}{\partial x}=\frac{1}{S c} \frac{\partial\left(\psi, \nabla^{2} \psi\right)}{\partial(x, z)}} \\
-\left(1+f \epsilon^{2} \delta_{1}\right) \frac{\partial \psi}{\partial x}+\left[\epsilon^{2} \frac{\partial}{\partial \tau}-\nabla^{2}\right] S=\frac{\partial(\psi, S)}{\partial(x, z)}
\end{gathered}
$$

Eqs. (17) and (18) can be written in matrix form as

$$
\begin{aligned}
& {\left[\begin{array}{cc}
\frac{\epsilon^{2}}{S c} \frac{\partial}{\partial \tau}\left(\nabla^{2}\right)-\nabla^{4}+H a^{2} \nabla^{2} & -R a s \frac{\partial}{\partial x} \\
-\left(1+f \epsilon^{2} \delta_{1}\right) \frac{\partial}{\partial x} & \epsilon^{2} \frac{\partial}{\partial s}-\nabla^{2}
\end{array}\right]\left[\begin{array}{l}
\psi \\
S
\end{array}\right]} \\
& =\left[\begin{array}{c}
\frac{1}{S c} \frac{\partial\left(\psi, \nabla^{2} \psi\right)}{\partial(x, z)} \\
\frac{\partial(\psi, S)}{\partial(x, z)}
\end{array}\right]
\end{aligned}
$$

We introduce a small perturbation parameter $\epsilon$ that shows deviation from the critical state of convective mass transfer, then the variable for a weakly nonlinear state may be expanded as power series of $\epsilon$ as in Eq. (19)

$$
\left.\begin{array}{l}
\psi=\epsilon \psi_{1}+\epsilon^{2} \psi_{2}+\ldots \\
S=\epsilon S_{1}+\epsilon^{2} S_{2}+\ldots \\
\operatorname{Ras}=\operatorname{Ras}_{0}+\epsilon^{2} \operatorname{Ras}_{2}+\ldots
\end{array}\right\}
$$

where $R a s_{0}$ is the critical solute Rayleigh number at which convective mass transfer sets without modulation, $\epsilon$ is the expansion parameter.

Now substituting Eq. (20) in Eq. (19) and comparing like powers of $\epsilon$ on both sides we get solution of different orders:

Solution for the first order case, we have

$$
\left[\begin{array}{cc}
-\nabla^{4}+H a^{2} \nabla^{2} & -\operatorname{Ras}_{0} \frac{\partial}{\partial x} \\
-\frac{\partial}{\partial x} & -\nabla^{2}
\end{array}\right]\left[\begin{array}{l}
\psi_{1} \\
S_{1}
\end{array}\right]=\left[\begin{array}{l}
0 \\
0
\end{array}\right]
$$

The solution of the lowest order system, can be written as

$$
\left.\begin{array}{l}
\psi_{1}=A(\tau) \sin \left(\kappa_{c} x\right) \cdot \sin (\pi z) \\
S_{1}=\frac{\kappa_{c}}{\delta^{2}} A(\tau) \cos \left(\kappa_{c} x\right) \cdot \sin (\pi z)
\end{array}\right\}
$$

where $\delta^{2}=\kappa_{c}^{2}+\pi^{2}$ is the total wave number. The expression of the critical solute Rayleigh number can be obtained from Eq. (21) and is given by

$$
\operatorname{Ras}_{0}=\frac{\delta^{6}+H a^{2} \delta^{4}}{\kappa_{c}^{2}}
$$

which is a similar result obtained by Gupta et al. [39] analogous to thermal convection for Couple stress parameter $C=0$. When $\mathrm{Ha}=0$, it recovers the result obtained by Chandrasekhar [4] analogous to thermal convection for Chandrasekhar number $Q=0$.

\subsection{Amplitude Equation (Ginzburg-Landau equation for mass transport of stationary Instability)}

At second order, we have

$$
\left[\begin{array}{cc}
-\nabla^{4}+H a^{2} \nabla^{2} & -\operatorname{Ras}_{0} \frac{\partial}{\partial x} \\
-\frac{\partial}{\partial x} & -\nabla^{2}
\end{array}\right]\left[\begin{array}{l}
\psi_{2} \\
S_{2}
\end{array}\right]=\left[\begin{array}{l}
R_{21} \\
R_{22}
\end{array}\right]
$$

where

$$
\begin{gathered}
R_{21}=\frac{1}{S c}\left[\frac{\partial \psi_{1}}{\partial x} \frac{\partial}{\partial z}\left(\nabla^{2} \psi_{1}\right)-\frac{\partial \psi_{1}}{\partial z} \frac{\partial}{\partial x}\left(\nabla^{2} \psi_{1}\right)\right] \\
R_{22}=\frac{\partial \psi_{1}}{\partial x} \frac{\partial S_{1}}{\partial z}-\frac{\partial \psi_{1}}{\partial z} \frac{\partial S_{1}}{\partial x}
\end{gathered}
$$

The solution of the second order system can be written as

$$
\left.\begin{array}{l}
\psi_{2}=0 \\
S_{2}=\frac{\kappa_{c}^{2}}{8 \pi \delta^{2}} A^{2}(\tau) \sin (2 \pi z)
\end{array}\right\}
$$

and we get

$$
\left.\begin{array}{l}
R_{21}=0 \\
R_{22}=\frac{\kappa_{c}^{2} A^{2}(\tau)}{2 \delta^{2}} \pi \sin (2 \pi z)
\end{array}\right\}
$$

Solution for the third order case we have

$$
\left[\begin{array}{cc}
-\nabla^{4}+H a^{2} \nabla^{2} & -\operatorname{Ras}_{0} \frac{\partial}{\partial x} \\
-\frac{\partial}{\partial x} & -\nabla^{2}
\end{array}\right]\left[\begin{array}{l}
\psi_{3} \\
S_{3}
\end{array}\right]=\left[\begin{array}{l}
R_{31} \\
R_{32}
\end{array}\right]
$$

where,

$$
R_{31}=\left[\frac{\delta^{2}}{S c} \frac{d A(\tau)}{d \tau}-\frac{\kappa_{c}^{2}}{\delta^{2}} A(\tau) \operatorname{Ras}_{2}\right] \sin \left(\kappa_{c} X\right) \sin (\pi z)
$$

and

$$
\begin{aligned}
& R_{32}=\left[-\frac{\kappa_{c}}{\delta^{2}} \frac{d A(\tau)}{d \tau}+\frac{\kappa_{c}^{3}}{4 \delta^{2}} A^{3}(\tau) \cos (2 \pi z)+\delta_{1} \kappa_{c} f A(\tau)\right] \\
& \cos \left(\kappa_{c} x\right) \sin (\pi z)
\end{aligned}
$$


Now, using the solvability condition for the third order solution and we get Ginzburg-Landau equation for stationary instability in the form

$$
\left(\frac{1}{S c\left(H a^{2}+\delta^{2}\right)}+\frac{1}{\delta^{2}}\right) \frac{d A(\tau)}{d \tau}-F(\tau) A(\tau)+\frac{\kappa_{c}^{2}}{8 \delta^{2}} A^{3}(\tau)=0
$$

where $F(\tau)=R^{\star}+2 \delta_{1} F(z, t)$ when $F(z, t)=$ $\int_{0}^{1} f \sin ^{2}(\pi z) d z \quad$ and $\quad R^{\star}=\frac{\text { Ras }_{2}}{\text { Ras }}$.

The horizontal local Sherwood number Sh (which measures rate of mass transport) is given by

$$
S h=1+\frac{\kappa_{c}^{2}}{4 \delta^{2}} A^{2}(\tau)
$$

\section{Analytical solution for unmodulated case}

In the case of unmodulated system, the Ginzburg- Landau equation obtained in Eq. (31) can be written as

$$
A_{1} A_{u}^{\prime}(\tau)-A_{2} A_{u}(\tau)+A_{3} A_{u}^{3}(\tau)=0,
$$

where $A_{u}(\tau)$ is amplitude of convective mass transport for unmodulated case. The coefficients $A_{1}, A_{2}$ and $A_{3}$ are given by

$A_{1}=\frac{1}{S c\left(H a^{2}+\delta^{2}\right)}+\frac{1}{\delta^{2}}, \quad A_{2}=\frac{\operatorname{Ras}_{2}}{\operatorname{Ras}_{0}} \quad$ and $\quad A_{3}=\frac{k_{c}^{2}}{8 \delta^{2}}$. The solution of Eq. (33) is given by

$$
A_{u}(\tau)=\frac{1}{\sqrt{\frac{A_{2}}{2 A_{3}}+C_{1} \exp \left[\frac{-2 A_{2}}{A_{1}}\right]}},
$$

where $C_{1}$ is a parameter and can be obtained by suitable initial conditions. The horizontal local Sherwood number for unmodulated case can be obtained by using the value of $A_{u}(\tau)$ in the place of $A(\tau)$ in Eq. (32).

\section{Time-periodic Gravity Modulation}

In this section we study the effect of Gravity modulation on the onset convection under the influence of timeperiodically varying gravity force $g \equiv(0,0,-g(t))$ acting on it, where $g(t)=g_{0}\left(1+\epsilon^{2} \delta_{2} \cos \Omega t\right)$ with $g_{0}$ the constant gravity in an otherwise unmodulated system, $\epsilon$ the small amplitude of modulation, $\omega$ the frequency of modulation and $\tau$ the time. A uniform adverse concentration gradient $\Delta S=\left(S_{l}-S_{u}\right)$ where $S_{l}<S_{u}$ is maintained between the lower and upper boundary.

At the third order solution, we obtained the following ex- pressions as obtained in Eqs. (29) and (30)

$$
\begin{gathered}
R_{31}=\left[\frac{\delta^{2}}{S c} \frac{d A(\tau)}{d \tau}-\left[\operatorname{Ras}_{2}+\operatorname{Ras}_{0} \delta_{2} \cos (\omega \tau)\right] \frac{\kappa_{c}^{2}}{\delta^{2}}\right] \\
\sin \left(\kappa_{c} x\right) \sin (\pi z) \\
R_{32}=\left[-\frac{\kappa_{c}}{\delta^{2}} \frac{d A(\tau)}{d \tau}+\frac{\kappa_{c}^{3}}{4 \delta^{2}} A^{3}(\tau) \cos (2 \pi z)\right] \cos \left(\kappa_{c} x\right) \sin (\pi z)
\end{gathered}
$$

Now by Eqs. (29), (30), (35), (36) and solve, we get Ginzburg-Landau equation for stationary instability in the form

$$
\left(\frac{1}{S c\left(H a^{2}+\delta^{2}\right)}-\frac{1}{\delta^{2}}\right) \frac{d A(\tau)}{d \tau}-F(\tau) A(\tau)+\frac{\kappa_{c}^{2}}{8 \delta^{2}} A^{3}(\tau)=0
$$

where $F(\tau)=R^{\star}+\delta_{2} \cos (\omega \tau)$

\section{Results and Discussion}

In this paper, we study the combined effect of concentration and gravity modulation on mass transport of a Newtonian fluid with an applied magnetic field. A weakly nonlinear stability analysis has been performed to investigate the effect of concentration/gravity modulation on mass transport. The effect of modulations on the system has been assumed to be of order $O\left(\epsilon^{2}\right)$. Means, we consider only small amplitude modulations. Such assumption will help us in obtaining the amplitude equation of diffusion in rather simple and elegant manner and is much easier to obtain than in the case of the Lorenz model(Chaotic system). It is important to make nonlinear stability analysis if one wants to study mass transport because linear stability analysis does not provide any information about mass transport. Linear stability analysis established the stability of the system for different parameters appeared in the model.

The parameters that appears in present study and influence the mass transport are $S c, H a, \delta_{1}, \delta_{2}$ and $\omega$. The first is related to the property of the fluid and rest concern the external mechanism that is Hartmann number $\mathrm{Ha}$, concentration and gravity modulation for controlling the mass transport. The values of amplitude of modulations ( $\delta_{1}$ and $\delta_{2}$ corresponding to concentration and gravity modulation respectively) are considered to be between 0 and 0.8 since we are studying the effect of small amplitude modulation on the mass transport. Further, as the effect of low frequencies on diffusion in solute concentration as well as the mass transport is maximum; therefore the modulations of concentration and gravity are assumed to be of low frequency. For all numerical computations the common value of the parameters are taken as 
$\delta_{1}=0.5, \delta_{2}=0.5, \omega=2, H a=2$ and $S c=0.05$ (In present paper, we have assumed that diffusion of solute concentration is high compared to diffusion in linear momentum, so the range of $S c$ has been chosen to be less than 1.).

The effect of concentration modulation on mass transport is depicted in Figs. 1-3, where graphs for the Sherwood number $S h$ are drawn with respect to slow time $\tau$. From these figures, it found that the effect of concentration modulation on mass transport is destabilizing and so that Sherwood number is more in the modulated case than in the absence of modulation. In Fig. 1, the variation of Sherwood number $S h$ with slow time performed for in-phase modulation (IPM) at different values of chosen parameters. In Fig. 1(a) and 1(b), we have shown the effect of amplitude of concentration modulation $\left(\delta_{1}\right)$ and frequency of concentration modulation $(\omega)$ respectively on Sherwood number (Sh). We can see from the Fig.1(a) and 1(b) that on increasing the value of $\delta_{1}$ and $\omega$, the value of $S h$ does not change, it means that amplitude of modulation $\left(\delta_{1}\right)$ and frequency of modulation $(\omega)$ has negligible effect on mass transport, which is a similar result obtained by Gupta et al. [41] and Gupta [42] for frequency of modulation and amplitude of modulation in case of in-phase concentration modulation. From Fig.1(c) and 1(d), we examine the effect of Hartmann number $(\mathrm{Ha})$ and Schmidt number $(\mathrm{Sc})$ on Sherwood number $(S h)$ respectively. It observed that diffusion takes place more rapidly for increasing value of Hartmann number as well as for Schmidt number. However, the effect of $\mathrm{Ha}$ and $S c$ on mass transport diminish at higher values of time $\tau$.

Fig. 2 displays the effect of chosen parameters on mass transport for out-phase modulation (OPM). In Fig.2(a), we examine the effect of amplitude of concentration modulation $\left(\delta_{1}\right)$ on Sherwood number $(S h)$. We observe that on the increase the value of an amplitude of concentration modulation $\left(\delta_{1}\right)$ increases the value of Sherwood number $(S h)$, which indicates that diffusion occurs and hence the rate of mass transfer increases. It means that effect of an amplitude of modulation on mass transport is destabilizing, a similar result investigated by Gupta et al. [41] for out phase concentration modulation. Effect of frequency of concentration modulation $(\omega)$ on the system is stabilizing (see fig.2(b)) as mass transport decreases on increasing the value of the frequency of concentration modulation. From Fig. 2(c) illustrate the effect of Hartmann number on mass transport rate in the case of out-phase modulation (OPM) while other parameters at their common values. As $\mathrm{Ha}$ increase the values of $S h$ increases. We know that Lorentz force opposes the fluid motion but we find that mass transport is still in progress because of the concentration at upper plate is much heavier that the lower plate. The effect

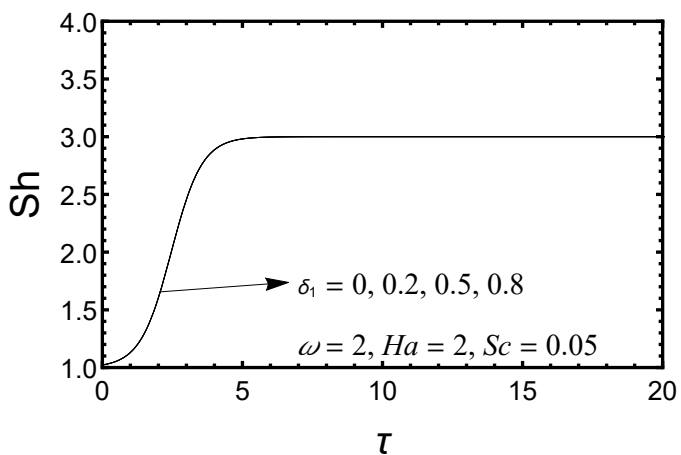

(a)

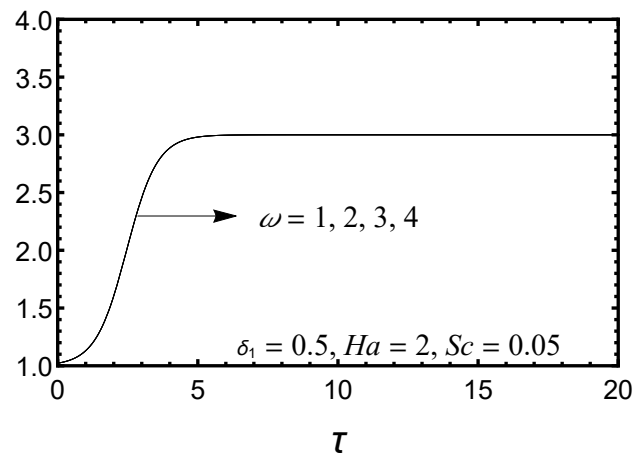

(b)

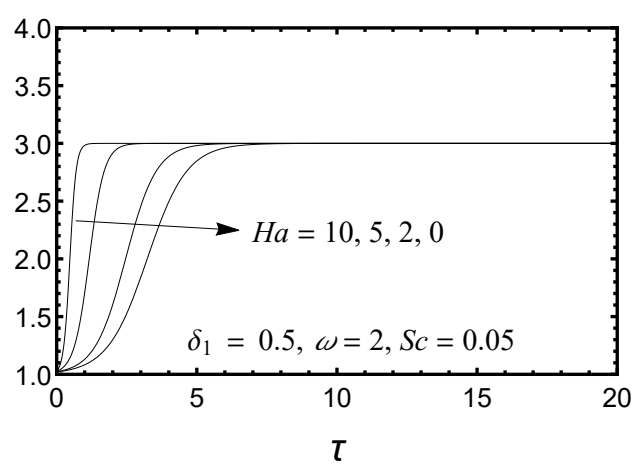

(c)

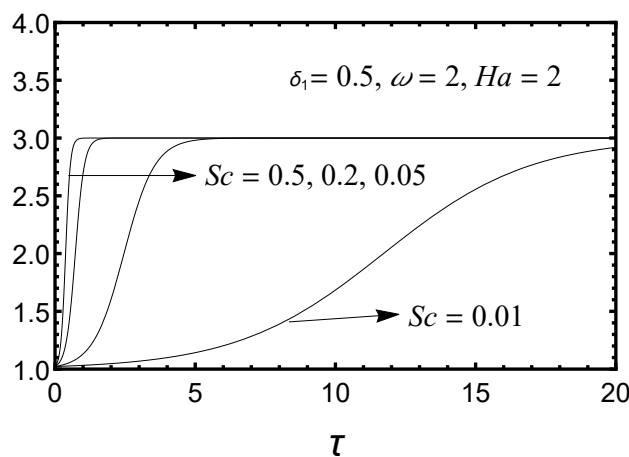

(d)

Fig. 1: Variation of Sherwood number Sh with time $\tau$ for in phase concentration modulation for different value of (a) $\delta_{1}$, (b) $\omega$, (c) $\mathrm{Ha}$, (d) $S c$. 


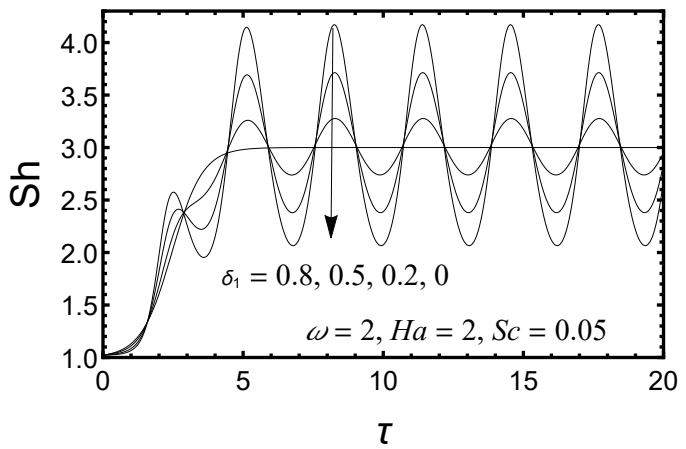

(a)

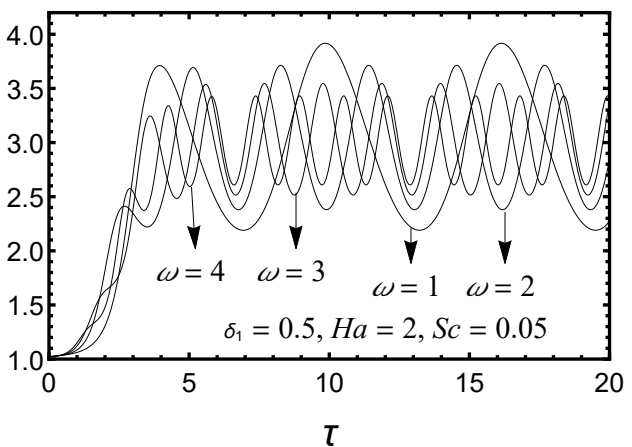

(b)

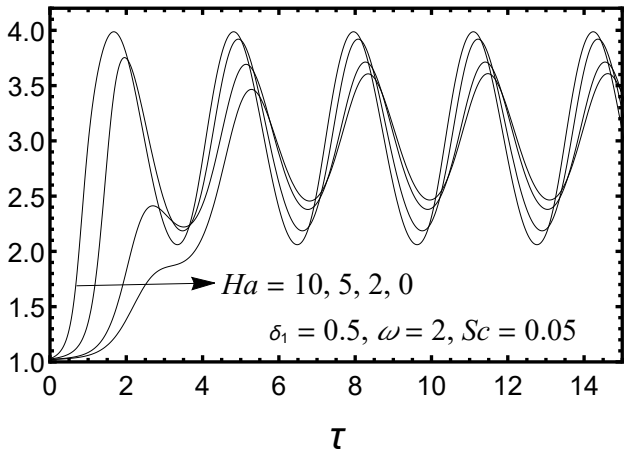

(c)

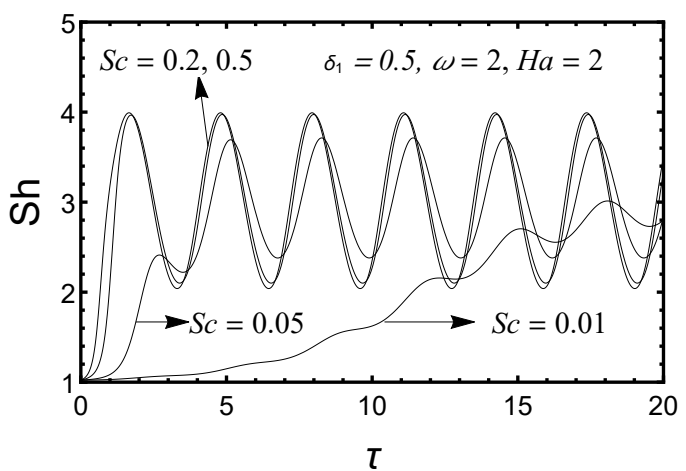

(d)

Fig. 2: Variation of Sherwood number Sh with time $\tau$ for out phase concentration modulation for different value of (a) $\delta_{1}$, (b) $\omega$, (c) $H a$, (d) $S c$.

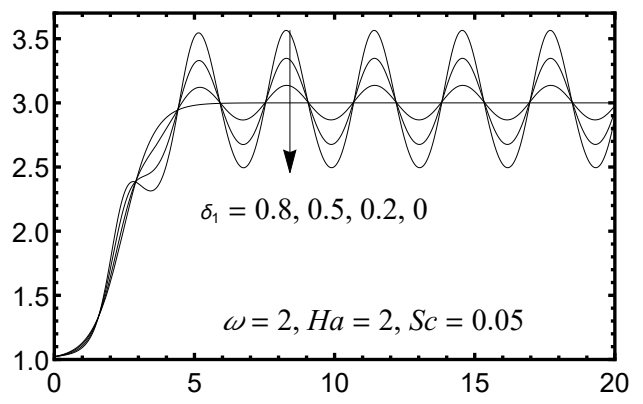

(a)

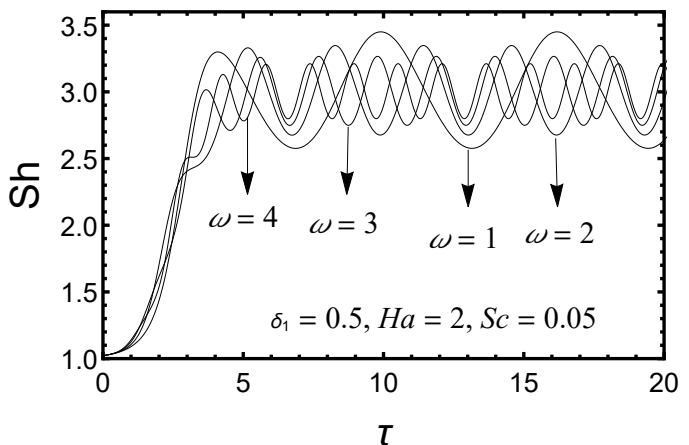

(b)

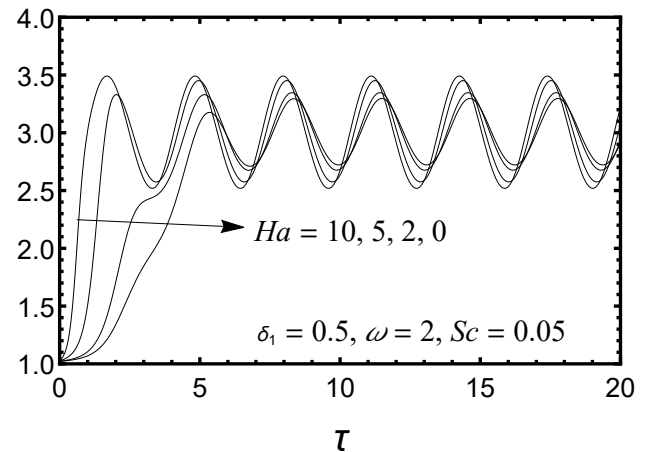

(c)

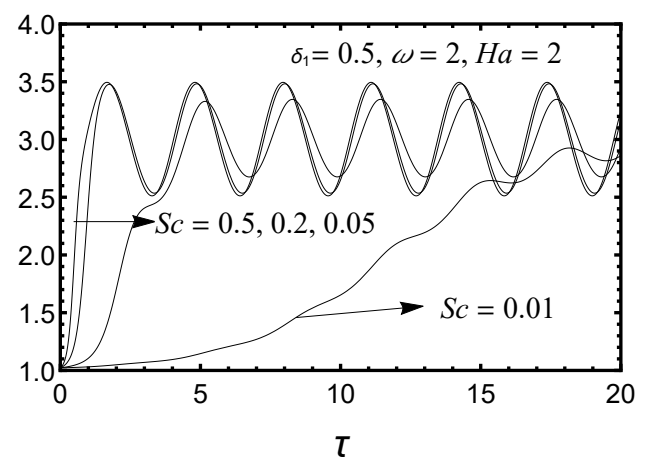

(d)

Fig. 3: Variation of Sherwood number Sh with time $\tau$ for only upper wall concentration is modulated while the lower one is held at constant concentration $(\varphi=-i \propto)$ for different value of (a) $\delta_{1}$, (b) $\omega$, (c) $H a$, (d)Sc. 


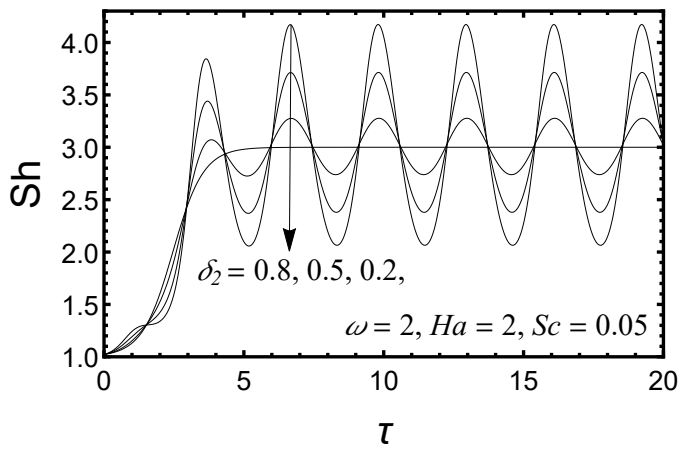

(a)

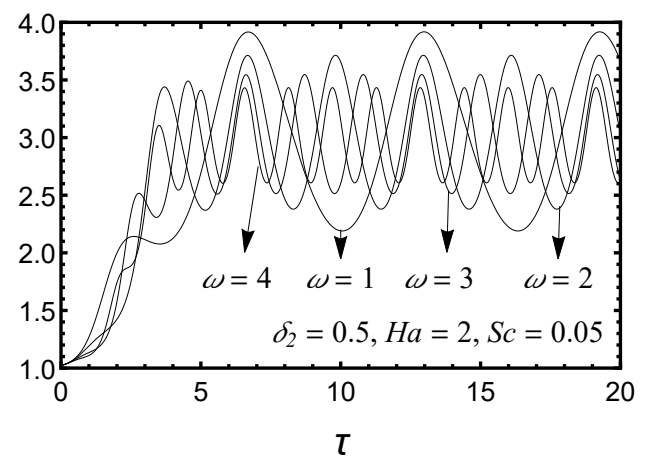

(b)

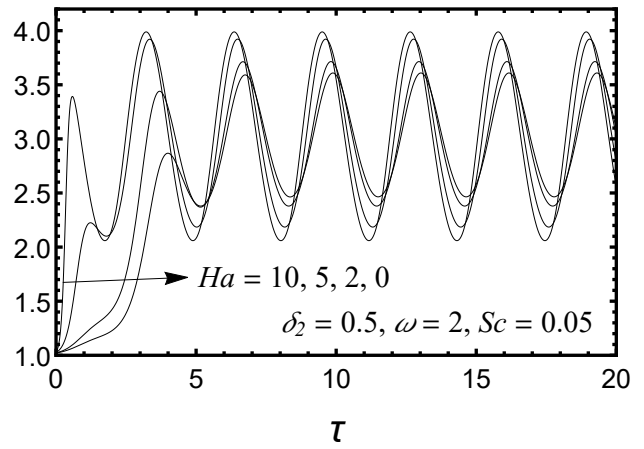

(c)

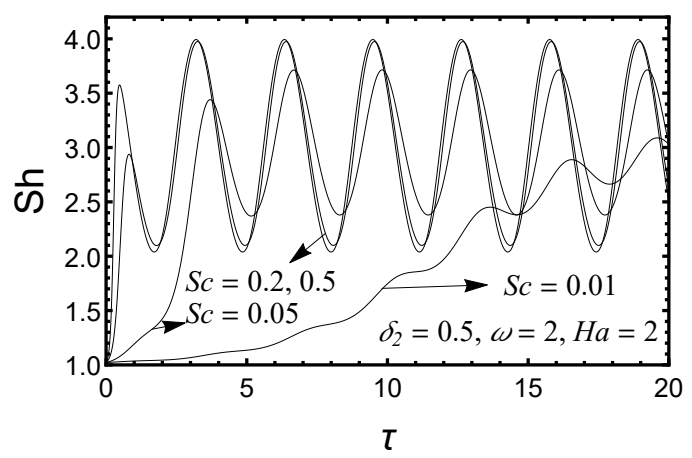

(d)

Fig. 4: Variation of Sherwood number Sh with time $\tau$ for gravity modulation for different value of (a) $\delta_{2}$, (b) $\omega$, (c) $H a$, (d) $S c$. of Schmidt number $(S c)$ on Sherwood number $(S h)$ in the case of out-phase modulation (OPM) while other parameters at their common values is illustrated in Fig. 2(d). With an increase in $S c$ from 0.01 through 0.05 to 0.2 , there is clear boosts in Sherwood number i.e. mass transport is strongly accelerated. It is evident that the increase in $S c$ concentration diffusivity decreases or kinematic viscosity increases in both the cases linear momentum increases and hence mass transport increases. From figs. 2(c) and 2(d), indicates that the effect of these parameters is to advance the diffusion, i.e., a rate of mass transfer increases. Hence, Hartmann number $\mathrm{Ha}$ and Schmidt number $\mathrm{Sc}$ has destabilizing effect for out phase modulation.

Fig. 3 depict the fact that rate of mass transport (Sh) in the case of upper plate modulation (UPM) variations with $\delta_{1}, \omega, H a$ and $S c$ are similar to what was found in Figs. 2(a)-(d). The results on $S h$ in Figs. 2 and 3 are as

$$
S h^{\mathrm{IPM}}<S h^{\mathrm{UPM}}<S h^{\mathrm{OPM}}
$$

Fig.4 describes the effect of gravity modulation for different parameters on mass transport rate. From Fig. 4(a), we have established the effect of an amplitude of gravity modulation $\delta_{2}$ on the rate of mass transfer (Sh). It found that amplitude of modulation $\delta_{2}$ has a destabilizing effect on the system as mass transport increases on increasing value of an amplitude of modulation. Fig. 4(b) shows that rate of mass transfer $(S h)$ decreases when we increase the value of the frequency of the gravity modulation $(\omega)$, i.e., a frequency of gravity modulation has to stabilize effect on the system. In Fig. 4(c), we depict the variation of $S h$ for different values of $H a$. It shows that diffusion takes place more rapidly with time when we increase the value of Hartmann number, and also it increases the rate of mass transfer. So, Hartmann number has the destabilizing effect. From Fig. 4(d), we found that increasing value of Schmidt number $(S c)$ increases the rate of mass transport and hence $S c$ destabilizes the system, a similar result were found by Gupta et al. [41].

\section{Conclusions}

In this paper, we demonstrated weakly nonlinear analysis of mass transport by perturbation technique to obtain a closed form expression for the problem of weakly nonlinear mass transport of the Newtonian fluid of an incompressible and electrically conducting viscous fluid within two infinite horizontal fluid layer. A uniform magnetic field applied perpendicular to both the plates and salted from above with subjected to an imposed 
time-periodic boundary concentration (ITBC) or gravity modulation (ITGM). The results obtained in this study are as follows:

1. The Hartmann number and the Schmidt number both have destabilized effect on the system.

2. The effect of increasing value of $\omega$ is to decrease the value Sherwood number.

3. The effect of increasing amplitude of modulation is to increase the value of mass transfer in both cases (concentration modulation and gravity modulation).

The results of this work for concentration modulation and gravity modulation can be summarized from the Figs. 1-4 as follows.

$$
\begin{array}{ll}
\text { 1. } & S h^{I P M}<S h^{U P M}<S h^{O P M} \\
\text { 2. } & S h /_{\delta_{1,2}=0}<S h /_{\delta_{1,2}=0.2}<S h /_{\delta_{1,2}=0.5}<S h /_{\delta_{1,2}=0.8} \\
\text { 3. } & S h /_{\omega=1}>S h /_{\omega=2}>S h /_{\omega=3}>S h /_{\omega=4} \\
\text { 4. } & S h /_{H a=0}<S h /_{H a=2}<S h /_{H a=5}<S h /_{H a=10} \\
\text { 5. } & S h /_{S c=0.01}<S h /_{S c=0.05}<S h /_{S c=0.2}<S h /_{S c=0.5}
\end{array}
$$

\section{Note}

Conflict of Interest: The authors declare that they have no conflict of interest.

Acknowledgement: All the authors would like to express our thanks to the anonymous editor and referees for their very expertise remarks and kind suggestions to improve the quality of manuscript.

\section{Nomenclature}

\begin{tabular}{ll}
\hline & Latin Symbols \\
$A$ & Amplitude of stream function \\
$B_{0}$ & Applied magnetic field \\
$g$ & Gravitational acceleration \\
$g_{0}$ & Reference value of gravitational acceleration \\
$H a$ & Hartman number \\
$\mathrm{p}$ & Atmospheric pressure \\
$\vec{q}$ & Velocity field $(u, 0, w)$ \\
$R a s$ & Solute Rayleigh number \\
$S$ & Solute Concentration \\
$S c$ & Schmidt number \\
$S h$ & Sherwood number \\
$t$ & Time \\
& Greek Symbols \\
$\beta_{S}$ & Coefficient of concentration expansion \\
$\kappa_{S}$ & Concentration diffusivity \\
$\delta_{1}$ & Amplitude of concentration modulation \\
\hline
\end{tabular}

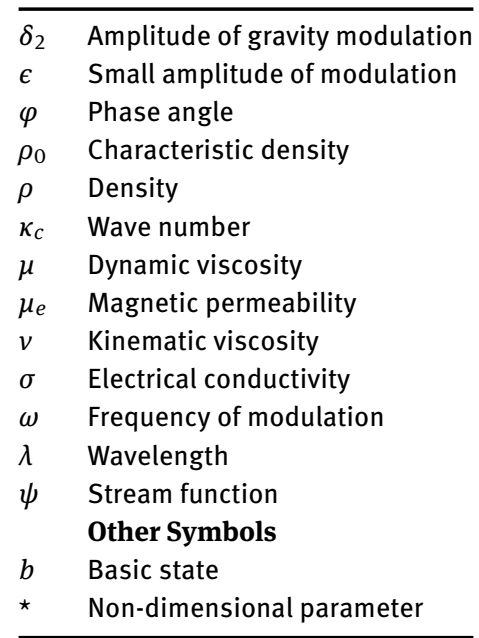

\section{References}

[1] Lewis W K, Whitman W G. Principles of Gas Absorption. Ind. Eng. Chem. 16, 1215-1220, 1924.

[2] Sherwood T K. Absorption and extraction. 1st Ed., Mcgraw-Hill Book Co., 1937.

[3] Bird R B, Steward W E, Lightfoot E N. Transport phenomena. 2nd Ed., Wiley, Inc, 1960.

[4] Chandrasekhar S. Hydrodynamic and hydromagnetic stability. Oxford University Press, 1961.

[5] Bejan A. Convection heat transfer. 2nd Ed., Wiley, New York, 1995.

[6] Donnelly R J. Experiments on the Stability of Viscous Flow Between Rotating Cylinder. III. Enhancement of Stability by Modulation. Proc Roy Soc A 1964, 281, 130-139.

[7] Venezian G. Effect of modulation on the onset of thermal convection. J Fluid Mech 1969, 35(2), 243-254.

[8] Rosenblat S, Herbert D M. Low frequency modulation of thermal instability. J Fluid Mech 1970, 43, 385-389.

[9] Davis S H. The stability of time periodic flow. Ann Rev Fluid Mech 1976, 8, 57-74.

[10] Rosenblat S, Tannaka G A. Modulation of thermal convection instability. Phys Fluid 1971, 14, 1319-1322.

[11] Roppo M N, Davis S H, Rosenblat S. Bénard convection with time-periodic heating. Phys Fluids 1984, 27, 796-803.

[12] Gershuni G Z, Zhukhovitskii E M. On parametric excitation of convective instability. J Appl Math Mech 1963, 27, 1197-1204.

[13] Yih C S, Li C H. Instability of unsteady flows or configurations. II: convective instability. J Fluid Mech 1972, 54, 143-152.

[14] Finucane R G, Kelly R E. Onset of instability in a fluid layer heated sinusoidally from below. Int J Heat Mass Transf 1976, 19, 71-85.

[15] Aniss S, Souhar M, Belhaq M. Asymptotic study of the convective parametric instability in Hele-Shaw cell. Phys Fluids 2000, 12, 262-268.

[16] Bhatia P K, Bhadauria B S. Effect of modulation on thermal convection instability. Z Naturforsch 2000, 55a, 957-966.

[17] Bhatia P K, Bhadauria B S. Effect of low-frequency modulation on thermal convection instability. Z Naturforsch 2001, 56a, 509-522. 
[18] Bhadauria B S, Bhatia P K. Time-periodic heating of RayleighBénard convection. Phys Scripta 2002, 66, 59-65.

[19] Bhaduria B S, Siddheshwar P G, Singh A K, Gupta V K. A local nonlinear stability analysis of modulated double diffusive stationary convection in a couple stress liquid. J App Fluid Mech 2015, 9, 1255-1264.

[20] Kumar A, Vanita, Gupta V K. Study of heat and mass transport in couple stress liquid under G-jitter effect. Ain Shams Engg J, Article in press, http://dx.doi.org/10.1016/j.asej.2016.05.003.

[21] Gersho P M, Sani R L. The effect of gravity modulation on the stability of a heated fluid layer. J Fluid Mech 1970, 40, 783806.

[22] Gershuni G Z, Zhukhovitskii E M, lurkov I S. On convective stability in the presence of periodically varying parameter. J Appl Math Mech 1970, 34, 442-452.

[23] Kamotini Y, Prasad A, Ostrac S. Thermal convection in enclosure due to vibrations aboard spacecraft. AIAAA J 1981, 19, 511-516.

[24] Biringen S, Danabasoglu G. Computation of convective flow with gravity modulation in rectangular cavities. J Thermophys 1900, 4, 357-365.

[25] Biringen S, Peltier L J. Numerical simulation of 3-D Bénard convection with gravitational modulation. Phys Fluid A 1990, 2, 754-764.

[26] Rogrers J R, Pesch W, Brausch O, Schatz M F. complex-ordered patterns in shaken Convection. Phys Rev E 2005, 71, 066214-118.

[27] Wadih M, Roux B. Natural convection in a long vertical cylinder under gravity modulation. J Fluid Mech 1988, 193, 391-415.

[28] Clever R, Schubert G, Busse F H. Two dimensional oscillatory convection in a gravitationally modulated fluid layer. J Fluid Mech 1993, 253, 663-680.

[29] Mohamed R A, Abo-Dahab S. M. Influence of chemical reaction and thermal radiation on the heat and mass transfer in MHD micropolar flow over a vertical moving porous plate in a porous medium with heat generation. Int J Thermal Sci 2009, 48, 1800-1813.

[30] Mohamed R A, Ibrahim A. Abbas, Abo-Dahab S M. Finite element analysis of hydromagnetic flow and heat transfer of a heat generation fluid over a surface embedded in a nonDarcian porous medium in the presence of chemical reaction. Commun Nonlinear Sci Numer Simulat 2009, 14, 1385-1395.

[31] Mohamed R A, Abo-Dahab S M, Nofal T A. Thermal Radiation and MHD Effects on Free Convective Flow of a Polar Fluid through a Porous Medium in the Presence of Internal Heat Generation and Chemical Reaction. Math Problems in Eng 2010, Article ID 804719, 1-27.

[32] Abdel-Nasser A 0, Abo-Dahab S M, Mohamed R A. Analytical Solution of Thermal Radiation and Chemical Reaction Effects on Unsteady MHD Convection through Porous Media with Heat Source/Sink. Math Problems in Eng 2011, Article ID 205181, 1-18.
[33] Abo-Dahab S M, Ibrahim H. El-Sirafy. On a Problem of Nonstationary 2D Motion of Micropolar Fluid in the Half-plane Subjected to a Uniform Magnetic Field when Normal Stresses and Tangential Velocities are Given on the Boundary. Int J Nonlinear Sci Numer Simul 2013, 14, 479-492.

[34] Abd-Alla A M, Abo-Dahab S M, Al-Simery R D. Effect of rotation on peristaltic flow of a micropolar fluid through a porous medium with an external magnetic field. J Magnetism and Magnetic Materials 2013, 348, 33-43.

[35] Kumar A, Singh A K. Unsteady MHD free convective flow past a semi-infinite vertical wall with induced magnetic field. Appl Math and Computation 2013, 222, 462-471.

[36] Abd-Alla A M, Abo-Dahab S M, El-Shahrany H D. Effects of rotation and initial stress on peristaltic transport of fourth grade fluid with heat transfer and induced magnetic field. J Magnetism and Magnetic Materials 2014, 349, 268-280.

[37] Kumar A, Singh A K, Chandran P, Sacheti N C. Effect of perpendicular magnetic field on free convection in a rectangular cavity. SQU Journal for Science 2015, 20, 49-59.

[38] Srivastava A K, Bhaduria B S, Gupta V K. Magneto-convection in an anisotropic porous layer with Soret effect. Int J NonLinear Mech 2012, 47, 426-438.

[39] Gupta V K, Prasad R, Singh A K. Effect of Magnetic field on chaos in couple stress liquid saturated in porous layer. Int J Engg Tech 2013, 5, 1-9.

[40] Swamy M S, Shivakumara I S, Naduvinamani N B. Effect of gravity modulation on electrothermal convection in a dielectric fluid saturated anisotropic porous layer. Journal of Heat Transfer 2013, 3 (136).

[41] Gupta V K, Kumar A, Singh A K. Analytical study of weakly nonlinear mass transfer in rotating fluid layer under timeperiodic concentration/gravity modulation, Int J of Non-Linear Mech. 2017, 97, 22-29.

[42] Gupta V K. Study of mass transport in rotating couple stress liquid under concentration modulation. Chinese Journal of Physics 2018, 56, 911-921.

[43] Coriell S R, Cordes M R, Boettinger W J, Sekerka R F. Convective and interfacial instabilities during unidirectional solidification of a binary alloy. J Cryst Growth 1980, 49, 13-28.

[44] Hurle D T J, Jakerman E, Wheeler A A. Hydrodynamic stability of the melt during solidification of a binary alloy. Physics of Fluids 1983, 26(3), 624-626.

[45] Wheeler A A, MsFadden G B, Murray T B, Coriell S R. Convective stability in the Rayleigh-Bénard and directional solidification problems: High-frequency gravity modulation. Phys Fluids A 1991, 3(12), 2847-2858.

[46] Siddheshwar P G, Bhadauria B S, Mishra P, Srivastava A K. Study of heat transport by stationary magneto-convection in a Newtonian liquid under temperature or gravity modulation using Ginzburg-Landau model. Int J Non-Linear Mechs 2012, 47, 418-425. 